\title{
Editorial \\ Inhibitory short synthetic oligodeoxynucleotides and lupus
} Zheng Liu ${ }^{1}$ and Anne Davidson²

\author{
${ }^{1}$ Department of Microbiology, Columbia University, New York, NY 10032 \\ ${ }^{2}$ Center for Autoimmunity and Musculoskeletal Diseases, Feinstein Institute for Medical Research, Manhasset, NY 11030, USA
}

Corresponding author: Anne Davidson, adavidson1@nshs.edu

Published: 26 June 2009

This article is online at http://arthritis-research.com/content/11/3/116

Arthritis Research \& Therapy 2009, 11:116 (doi:10.1186/ar2726)

(c) 2009 BioMed Central Ltd

See related research by Lenert et al., http://arthritis-research.com/content/11/3/R79

\begin{abstract}
B cells and antigen-presenting cells express a group of intracellular Toll-like receptors (TLRs) that recognize nucleic acids and can be accessed only when apoptotic debris or immune complexes are internalized by B-cell receptors or Fc receptors. This results in rapid cell activation and release of inflammatory mediators that perpetuate the autoantibody response. TLR-7 and TLR-9 are required to generate autoantibodies to RNA and DNA, respectively. Synthetic oligodeoxynucleotides that inhibit the activity of these intracellular TLRs attenuate systemic lupus erythematosus in mouse models and may be of therapeutic benefit in human systemic lupus erythematosus.
\end{abstract}

Although clonal expansion of autoreactive B and T lymphocytes is a hallmark of autoimmune disease, innate immunity also plays an important role in the initiation and perpetuation of autoimmunity. Toll-like receptors (TLRs) are innate immune receptors that recognize structural components of microorganisms and trigger an immediate pro-inflammatory response. A group of intracellular TLRs specifically recognizes nucleic acids including double-stranded RNA (TLR-3), single-stranded RNA (TLR-7/8), and CpG-rich DNA (TLR-9) [1] (for review [2]). Once engaged, these TLRs act rapidly via adaptor proteins to induce transcription factors for type I IFNs and other pro-inflammatory mediators.

Access to intracellular TLRs requires that nucleic acid antigens reach the right subcellular compartment. Immune complexes containing nucleic acids or opsonized apoptotic debris are internalized via $\mathrm{FC}_{\mathrm{C}}$ receptors or B-cell receptors (BCRs) into TLR-7/9 expressing dendritic cells and B cells, respectively [3]. Once these nucleic acid payloads enter cells they recruit TLR-containing endosomes to form an autophagosome, in which TLRs survey the internalized antigen [4]. TLR engagement in plasmacytoid dendritic cells induces type I IFN production [3], whereas TLR engagement in $\mathrm{B}$ cells increases BCR signaling and antibody production [4]. The interaction of TLRs, type I IFNs, and B-cell activating factor (BAFF) creates an amplification loop that may propagate the production of autoantibodies to nucleic acids in the absence of T-cell help (Figure 1).

Studies in knock-out animals have conclusively shown that the anti-RNA response requires TLR-7 whereas the anti-DNA response requires TLR-9, and that both responses require the key adaptor molecule MyD88 [5]. The importance of nucleic acid recognizing TLRs in the pathogenesis of systemic lupus erythematosus (SLE) has been further illustrated by studies showing that TLR-7 over-expression accelerates or initiates SLE in mice [6], whereas TLR-7 deficiency attenuates disease [5]. Although TLR-9 deficiency abrogates the anti-DNA response, it worsens the disease in some strains of mice $[5,7]$. This may be because TLR-9 negatively regulates the production of IFN- $\alpha$ in immature dendritic cells and the increased IFN- $\alpha$ drives the amplification loop shown in Figure 1; via TLR-7 upregulation, this results in selection of $B$ cells that secrete pathogenic anti-RNA antibodies.

Because expression of type I IFNs and BAFF is increased in SLE patients, intracellular TLRs, type I IFNs, and BAFF/APRIL (a proliferation ligand) are being intensely pursued as therapeutic targets in SLE. Targeting of intracellular TLRs was made possible by the discovery that short synthetic oligodeoxynucleotides (ODNs) on a nuclease-resistant phosphorothioate backbone can either stimulate or inhibit TLR activity. Inhibitory sequences for TLR-9 need GGG or GGGG sequences and most also contain CCT at the 5' end [8]. Inhibition of TLR-7 requires a phosphorothioate backbone but is much less dependent on the ODN sequence.

$\mathrm{BAFF}=\mathrm{B}$-cell activating factor; BCR = B-cell receptor; IFN = interferon; ODN = short synthetic oligodeoxynucleotide; SLE = systemic lupus erythematosus; $\mathrm{TLR}=$ Toll-like receptor. 
Figure 1

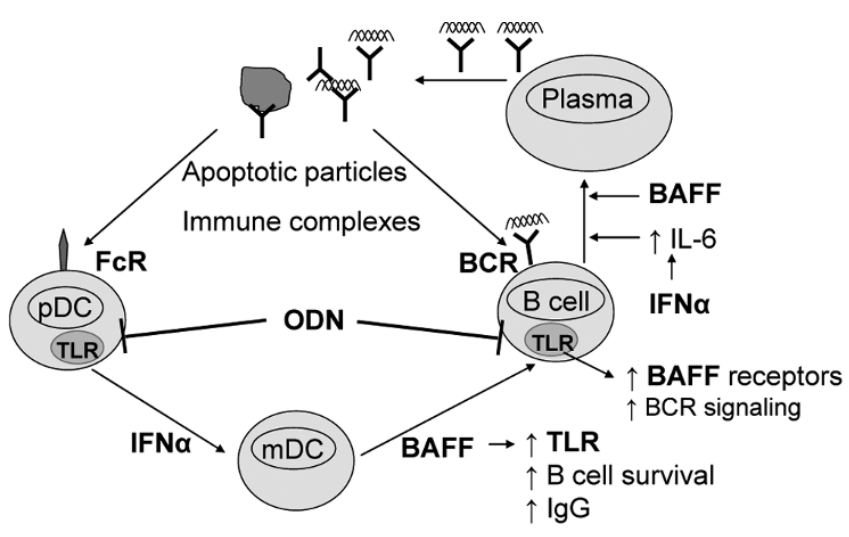

T-independent autoantibody production may be propagated by an amplification loop involving TLRs, IFN $\alpha$, and BAFF/APRIL. BAFF, B-cell activating factor; BCR, B-cell receptor; DC, dendritic cell; FcR, Fc receptor; IFN, interferon; IL, interleukin; $\mathrm{mDC}$, monocyte-derived dendritic cell; ODN, short synthetic oligodeoxynucleotide; pDC, plasmacytoid dendritic cell; SLE, systemic lupus erythematosus; TLR, Toll-like receptor.

Inhibitory ODNs are of two broad structural types. Linear (class B) ODNs inhibit both naïve B cells and professional antigen-presenting cells (including macrophages and dendritic cells), whereas ODNs with more complex secondary structure (class $\mathrm{R}$ ) inhibit antigen-presenting cells but have no effect on naïve $B$ cells [8]. Most reported ODNs inhibit both TLR-7 and TLR-9, but TLR-specific ODNs have also been generated. In vitro, inhibitory ODNs specific for TLR-7 or TLR-9 inhibit the stimulatory effects of RNA- and DNA-containing immune complexes, respectively, and ODNs specific for both TLRs inhibit the effects of both types of immune complexes [8]. In vivo, however, inhibition of TLR-7 alone is sufficient to attenuate formation of both anti-RNA and anti-DNA antibodies and to mitigate disease, especially at early stages; in the only published comparative study [9], inhibition of both TLR-7 and TLR-9 did not appear to have an enhanced effect on disease over inhibition of TLR-7 alone. Thus, for reasons of safety, blockade of TLR-9 alone is probably not a good therapeutic strategy in SLE.

The fine structural features and therapeutic efficacy of a set of inhibitory ODNs are the subject of a report by Lenert and coworkers published in this issue of Arthritis Research and Therapy [1]. Using ODNs that contain a palindrome and a linear segment of various lengths, the authors show that ODNs with progressively longer linear segments are increasingly capable of inhibiting TLR-9 signaling in naïve $B$ cells. They also show that the inability of class R ODNs to inhibit naïve B cells is bypassed when BCR signaling is present; the most likely explanation for this observation is that concomitant BCR stimulation promotes the formation of autophagosomes and thus allows the ODNs to reach the appropriate subcellular compartment [4]. Thus, if anti-nucleic acid autoantibodies are present, then both linear and complex ODNs should be able to access the TLR compartment in naïve $B$ cells in vivo. This is important because in models of TLR-7 over-expression, excess TLR-7 on B cells alone is sufficient to drive disease [10]. Surprisingly, the class $R$ (complex) ODN was more therapeutically efficacious than the class B ODN in lupus-prone mice, although the latter could target a wider range of cells in vitro. Despite the dual specificity of the ODN for TLR-7 and TLR-9, the anti-RNA response was inhibited much more effectively than the antiDNA response. Together, these findings suggest that caution is needed when using in vitro data if the aim is to obtain a reliable prediction of therapeutic efficacy of an ODN in vivo.

As inhibitory ODNs move toward clinical trials many questions remain. Will inhibitory ODNs be more effective, safer, or more specific than blockade of other components of the TLR/type I IFN/BAFF amplification loop? Will they synergize with other antagonists of the other components of the loop such as IFN- $\alpha$ or BAFF, or with antagonists of T-cell function? Mouse studies show that ODNs are most effective when used as a preventive therapy [2]. What will be the effect of ODN treatment if active inflammation is already present? Does the autoantibody specificity (DNA or RNA, or both) in individual patients matter? TLR-7 inhibitors do not necessarily inhibit TLR-8 that is expressed predominantly in human monocytes and myeloid dendritic cells and triggers a nuclear factor- $\kappa B$ driven pro-inflammatory response [11]. Does TLR-8, which is not functional in mouse, play a role in SLE pathogenesis in humans? Is there a way to optimize ODN structure and sequence so as to achieve maximal inhibition in vivo? Although some of these questions can be answered by more experiments in mouse models, carefully designed human clinical trials will be needed to evaluate the true potential of inhibitory ODNs as therapies for SLE.

\section{Competing interests}

The authors declare that they have no competing interests.

\section{Acknowledgements}

We acknowledge support from the NIH to AD (R01 AR 049938).

\section{References}

1. Lenert P, Yasuda K, Busconi L, Nelson P, Fleenor C, Ratnabalasuriar RS, Nagy PL, Ashman RL, Rifkin IR, Marshak-Rothstein A: DNA-like class $\mathrm{R}$ inhibitory oligonucleotides (INH-ODN) preferentially block autoantigen-induced $\mathrm{B}$-cell and dendritic cell activation in vitro and autoantibody production in lupus-prone MRL-Fas/pr/lpr mice in vivo. Arthritis Res Ther 2009, 11:R79.

2. Barrat FJ, Coffman RL: Development of TLR inhibitors for the treatment of autoimmune diseases. Immunol Rev 2008, 223: 271-283.

3. Gilliet M, Cao W, Liu YJ: Plasmacytoid dendritic cells: sensing nucleic acids in viral infection and autoimmune diseases. Nat Rev Immunol 2008, 8:594-606.

4. Chaturvedi A, Dorward D, Pierce SK: The B cell receptor governs the subcellular location of Toll-like receptor 9 leading to hyperresponses to DNA-containing antigens. Immunity 2008, 28:799-809.

5. Christensen SR, Shlomchik MJ: Regulation of lupus-related autoantibody production and clinical disease by Toll-like 
receptors. Semin Immunol 2007, 19:11-23.

6. Deane JA, Pisitkun P, Barrett RS, Feigenbaum L, Town T, Ward JM, Flavell RA, Bolland S: Control of toll-like receptor 7 expression is essential to restrict autoimmunity and dendritic cell proliferation. Immunity 2007, 27:801-810.

7. Ehlers M, Ravetch JV: Opposing effects of Toll-like receptor stimulation induce autoimmunity or tolerance. Trends Immunol 2007, 28:74-79.

8. Lenert PS: Targeting Toll-like receptor signaling in plasmacytoid dendritic cells and autoreactive $B$ cells as a therapy for lupus. Arthritis Res Ther 2006, 8:203.

9. Pawar RD, Ramanjaneyulu A, Kulkarni OP, Lech M, Segerer S, Anders HJ: Inhibition of Toll-like receptor-7 (TLR-7) or TLR-7 plus TLR-9 attenuates glomerulonephritis and lung injury in experimental lupus. J Am Soc Nephrol 2007, 18:1721-1731.

10. Fossati L, Sobel ES, Iwamoto M, Cohen PL, Eisenberg RA, Izui S: The Yaa gene-mediated acceleration of murine lupus: Yaa- $T$ cells from non-autoimmune mice collaborate with Yaa+ B cells to produce lupus autoantibodies in vivo. Eur J Immunol 1995, 25:3412-3417.

11. Iwasaki A, Medzhitov R: Toll-like receptor control of the adaptive immune responses. Nat Immunol 2004, 5:987-995. 